\title{
ISCHEMIC PRECONDITIONING DOES NOT ACUTELY IMPROVE LOAD-INSENSITIVE PARAMETERS OF CONTRACTILITY IN IN VIVO STUNNED PORCINE M YOCARDIUM
}

M. Salik Jahania, MD

Robert D. Lasley, PhD

Robert M. Mentzer, Jr, MD
Objective: Ischemic preconditioning has been shown to have no beneficial effect on segment shortening in in vivo regionally stunned myocardium. The purpose of this study was to determine whether ischemic preconditioning improves the recovery of postischemic ventricular function when contractility is assessed by load-insensitive measurements including end-systolic pressure length relations, preload recruitable stroke work, and preload recruitable stroke work area in in vivo regionally stunned porcine myocardium. Methods: Open chest, pentobarbital-anesthetized pigs were used. Regional ventricular function was monitored by measurements of segment shortening, stroke work, end systolic pressure length relations, preload recruitable stroke work, and preload recruitable stroke work area. The control group was submitted to 15 minutes of left anterior descending coronary artery occlusion and 3 hours of reperfusion. The preconditioned group underwent 2 cycles of 5 minute left anterior descending coronary artery occlusion and 10minute reperfusion before $\mathbf{1 5}$ minutes of occlusion. Results: There was no infarct in either group. The preconditioning protocol significantly depressed preischemic segment shortening but not regional stroke work. Ischemic preconditioning had no significant beneficial effect on regional stroke work, end-systolic pressure length relations, preload recruitable stroke work, or preload recruitable stroke work area. Conclusions: These results confirm that ischemic preconditioning does not ameliorate in vivo porcine myocardial stunning and indicate that ischemic preconditioning may have a limited cardioprotective role during cardiac operation. (J Thorac Cardiovasc Surg 1999;117:810-7) schemic preconditioning is the phenomenon whereby

1 or more brief coronary occlusion/reperfusion cycles before a prolonged occlusion significantly improves myocardial tolerance to ischemia. Since the observation by Murry and colleagues ${ }^{1}$ that ischemic preconditioning reduced infarct size, there have been numerous studies documenting the effects of preconditioning in several animal models and species. ${ }^{2}$ Ischemic precondi-

From the Department of Surgery, University of Kentucky College of Medicine, Lexington, Ky.

Supported by grant HL34579 from The National Heart, Lung, and Blood Institute.

Received for publication Aug 31, 1998; revisions requested Oct 30, 1998; revisions received Dec 4, 1998; accepted for publication Dec 4, 1998.

Address for reprints: M. Salik Jahania, MD, Department of Surgery, University of Kentucky College of Medicine, Albert B. Chandler Medical Center, MN 273 B, 800 Rose St, Lexington, KY 405360084.

Copyright (C) 1999 by Mosby, Inc.

$0022-5223 / 99 \$ 8.00+0 \quad \mathbf{1 2 / 1 / 9 6 4 0 5}$ tioning has been shown to have numerous beneficial metabolic effects such as reduced rates of high-energy phosphate catabolism and reduced accumulation of myocardial lactate and hydrogen ions during prolonged ischemia. ${ }^{3-5}$ There is also clinical evidence indicating human myocardium may be preconditioned during balloon angioplasty ${ }^{6}$ and cardiac operation. ${ }^{7,8}$

The role of ischemic preconditioning in attenuating reversible postischemic dysfunction, however, remains controversial. Although ischemic preconditioning consistently improves postischemic recovery of function in the isolated perfused rat heart, ${ }^{9-11}$ there are conflicting reports about its effects in the globally ischemic rabbit heart. ${ }^{12-17}$ In rabbit in vivo regional ischemia models, ischemic preconditioning has been shown to have no beneficial effect on postischemic regional function independent of infarct size reduction. ${ }^{18}$ In 2 in vivo large animal studies Ovize, ${ }^{19}$ Miyamae,${ }^{20}$ and their colleagues reported that ischemic preconditioning did not exert any beneficial effect on regional segment shorten- 
ing in canine and porcine myocardium. However in both studies, the substantial decrease in segment shortening, associated with the 5-minute preconditioning cycles, itself may have obscured any protective effect after the subsequent longer occlusion. Furthermore, assessment of regional ventricular function by segment shortening is preload and afterload dependent, which hinders its use as a sensitive measure of cardiac contractility. In contrast, load insensitive measures, such as the end-systolic pressure segment length relationship (ESPLR), preload recruitable stroke work (PRSW) and preload recruitable stroke work area (PRSWA) are more sensitive and reliable indicators of cardiac contractility in intact animals. ${ }^{21-24}$ Therefore the purpose of this study was to assess the effects of ischemic preconditioning on both load-sensitive and load-insensitive measurements of contractility in a porcine model of in vivo regional myocardial stunning.

\section{Methods}

All animals in this study received humane care according to the guidelines set forth in "The Principles of Laboratory Animal Care" formulated by the National Society for Medical Research and the "Guide for the Care and Use of Laboratory Animals" prepared by the Institute of Laboratory Animal Resources and published by the National Institutes of Health (NIH Publication No. 86-23, revised 1996). In addition, animals were used in accordance with the guidelines of the University of Kentucky Institutional Animal Care and Use Protocol.

Animal preparation. Farm pigs weighing 22 to $27 \mathrm{~kg}$ were used. Anesthesia was induced with ketamine $(20 \mathrm{mg} / \mathrm{kg}$, intramuscularly) and sodium pentobarbital $(15-18 \mathrm{mg} / \mathrm{kg}$, intravenously). Anesthesia was maintained with additional sodium pentobarbital $(1.5-2 \mathrm{mg} / \mathrm{kg}$, intravenously) every 15 minutes. Ventilation was maintained with a tracheotomy with a mixture of room air and $100 \%$ oxygen. Tidal volume, respiratory rate, and fraction of oxygen in inspired air were adjusted to maintain normal arterial blood gas and $\mathrm{pH}$ values. Core body temperature was monitored with an esophageal temperature probe and maintained with a heating pad between $37.0^{\circ} \mathrm{C}$ and $37.5^{\circ} \mathrm{C}$. Lactated Ringer's solution was administered through an ear vein or femoral vein, at 5 to 7 $\mathrm{mL} / \mathrm{kg} / \mathrm{min}$ after an initial bolus of 300 to $400 \mathrm{~mL}$. A femoral artery catheter was used to monitor arterial blood pressure and to obtain arterial blood gas samples.

The heart was exposed through a median sternotomy and suspended in a pericardial cradle. Left ventricular pressure (LVP) was measured with a 5F high-fidelity pressure-sensitive tip transducer (Millar Instruments, Houston, Tex) placed in the left ventricular cavity through the apex and secured with a purse-string suture. A segment of the left anterior descending coronary artery (LAD), distal to the origin of the first diagonal branch, was dissected free of surrounding tissue. The area at risk was delineated by a brief $(<20$-second $)$ occlusion of the LAD with a small vascular occluding clamp. A transit time perivascular flow probe (Transonic Systems Inc, Ithaca, NY) was placed around this segment to measure coronary blood flow.

Pairs of piezoelectric segment-shortening crystals (Crystal Biotech, Houston, Tex) were placed in the LAD and left circumflex coronary artery perfused beds to measure regional segment shortening by sonomicrometry. Crystals were placed in the mid-myocardium (approximately 4-6 $\mathrm{mm}$ deep) 5 to $15 \mathrm{~mm}$ apart and aligned in a manner such that the intercrystal axis was parallel to the direction of myocardial fiber shortening.

Experimental protocol. Animals in both groups were allowed a 30-minute stabilization period after all instrumentation was complete. A total of 19 pigs were randomized into 2 groups. The control group $(n=11)$ underwent a 15 -minute LAD occlusion and 3-hour reperfusion. The ischemic preconditioning group $(n=8)$ underwent 2 cycles of 5 -minute LAD occlusion and 10-minute reperfusion before the 15minute occlusion. All animals received heparin $(100 \mathrm{U} / \mathrm{kg}$ body weight, intravenously) before the coronary occlusion and lidocaine ( $2 \%$ solution; $2 \mathrm{mg} / \mathrm{kg}$, intravenous bolus) immediately before reperfusion. After 3 hours of reperfusion, the ischemic area at risk was determined by reoccluding the LAD and infusing a $0.5 \%$ Evans blue solution into the left ventricle while occluding the aorta. The area at risk was devoid of the Evans blue stain. The animals were killed while under deep anesthesia with an intracardiac bolus of $\mathrm{KCl}$, and the hearts were excised. Crystal placement in the ischemic and nonischemic beds was verified after excision of the heart.

Area at risk and infarct size measurement. The isolated left ventricles were cut in 4 slices of equal thickness in a plane parallel to the atrioventricular groove. Each slice was weighed and compressed between 2 transparent Plexiglas acrylic plates (Rohm \& Hass Company, Philadelphia, Pa) separated by a distance of $8 \mathrm{~mm}$ to achieve uniform thickness. The cross-sectional surface and ischemic areas of each slice were traced onto a transparency sheet. The slices were then incubated in a $1 \%$ triphenyltetrazolium chloride solution in phosphate-buffered saline solution at $37^{\circ} \mathrm{C}$ for 15 minutes. The presence of a brick-red stain indicated viable tissue although nonviable tissue (infarcted) remained pale. If any infarct was present, the tissue slices were again compressed between the Plexiglas acrylic plates and retraced. The areas on the tracings were quantified with a digitizer (Mustek 1200 III, parallel port scanner at $200 \mathrm{dpi}$; Mustek Incorporated, Irvine, Calif) and graphic analysis software (Sigma Scan Pro Automated Image Analysis Software; Jandel Scientific, SPSS Inc, San Rafael, Calif). The percent area at risk was calculated for each slice by dividing the area at risk by the total slice area. The sum of the areas at risk of all slices was divided by the sum of the areas of all slices to obtain the percentage of the left ventricle that was ischemic.

Data and statistical analysis. All hemodynamic and sonomicrometry signals were fed through a 32-bit analog digital converter into an online data acquisition computer with customized software (Augury; Coyote Bay Instruments, Manchester, NH). End-diastole was defined as the onset of 
Table I. Global ventricular function and hemodynamic data

\begin{tabular}{|c|c|c|c|c|c|c|c|c|c|c|}
\hline & \multicolumn{5}{|c|}{ Control group } & \multicolumn{5}{|c|}{ Ischemic preconditioning group } \\
\hline & $\begin{array}{c}M A P \\
(m m H g)\end{array}$ & $\begin{array}{c}H R \\
\text { (beats/min) }\end{array}$ & $\begin{array}{c}L V D P \\
(m m \mathrm{Hg})\end{array}$ & $\begin{array}{c}+d P / d t \\
(m m H g / s)\end{array}$ & $\begin{array}{c}C B F \\
(\mathrm{~mL} / \mathrm{min})\end{array}$ & $\begin{array}{c}M A P \\
(m m H g)\end{array}$ & $\begin{array}{c}H R \\
\text { (beats/min) }\end{array}$ & $\begin{array}{c}L V D P \\
(m m H g)\end{array}$ & $\begin{array}{c}+d P / d t \\
(m m ~ H g / s)\end{array}$ & $\begin{array}{c}C B F \\
(\mathrm{~mL} / \mathrm{min})\end{array}$ \\
\hline Baseline & $96 \pm 5$ & $107 \pm 9$ & $87 \pm 4$ & $1333 \pm 31$ & $20 \pm 1$ & $92 \pm 3$ & $103 \pm 6$ & $81 \pm 5$ & $1270 \pm 61$ & $21 \pm 1$ \\
\hline Ischemia & $93 \pm 7$ & $107 \pm 11$ & $78 \pm 3$ & $1203 \pm 38$ & 0 & $90 \pm 3$ & $105 \pm 7$ & $77 \pm 6$ & $1111 \pm 33$ & 0 \\
\hline 1 Hour & $94 \pm 6$ & $105 \pm 12$ & $83 \pm 5$ & $1151 \pm 43$ & $13 \pm 2^{*}$ & $86 \pm 3$ & $108 \pm 6$ & $81 \pm 2$ & $1036 \pm 54$ & $10 \pm 2 \dagger$ \\
\hline 2 Hours & $89 \pm 6$ & $108 \pm 11$ & $80 \pm 6$ & $1156 \pm 57$ & $12 \pm 2 \ddagger$ & $84 \pm 4$ & $113 \pm 7$ & $81 \pm 3$ & $1033 \pm 48$ & $11 \pm 2 \S$ \\
\hline 3 Hours & $86 \pm 6$ & $109 \pm 11$ & $78 \pm 3$ & $1144 \pm 51$ & $13 \pm 2^{*}$ & $82 \pm 4$ & $114 \pm 8$ & $77 \pm 5$ & $1043 \pm 48$ & $12 \pm 2 \mathrm{Il}$ \\
\hline
\end{tabular}

Values are expressed as mean \pm SEM.

$M A P$, Mean arterial pressure; $H R$, heart rate; $C B F$, coronary blood flow.

The baseline data for the ischemic preconditioning group represent data after the preconditioning protocol but before the onset of the 15-minute LAD occlusion.

$* P<.01$ versus baseline.

$\dagger P=.0002$ versus baseline.

$\ddagger P=.009$ versus baseline.

$\S P=.0003$ versus baseline.

$\| P=.0008$ versus baseline

positive rate of pressure rise $(+\mathrm{dP} / \mathrm{dt})$ and end-systole defined as $20 \mathrm{~ms}$ before peak $-\mathrm{dP} / \mathrm{dt}$. Segment shortening was defined as end-diastolic length (EDL)-end-systolic length (ESL), and percent segment shortening (\% SS) was calculated as (EDL $\mathrm{ESL} / \mathrm{EDL}) \times 100 \%$. All hemodynamic data were continuously displayed on a computer monitor. Stroke work (SW) was calculated by quantifying the area within the pressure-segment-length loops generated during each cardiac cycle.

ESPLR, PRSW, and PRSWA were generated from the segment length, and LVP data collected during brief (7-second) vena cava occlusions. The inferior vena cava was occluded by gradual tightening of a snare formed of umbilical tape around its supradiaphragmatic portion. During data acquisition ventilation was held at end expiration to avoid effects of varying venous return on preload. Slope of the ESPLR $\left(\mathrm{E}_{\mathrm{es}}\right)$ was calculated according to the methods of Aversano and colleagues, ${ }^{23}$ where end-systole during each cardiac cycle was defined as the point on the LVP-segment length loop that maximized the value of $\mathrm{E}_{\mathrm{es}}$. The segment length (x-axis) intercept at an LVP of $70 \mathrm{~mm} \mathrm{Hg}\left(\mathrm{L}_{70}\right)$ was derived with the slope and $\mathrm{x}$ - and $\mathrm{y}$-intercept data from the ESPLR. PRSW and PRSWA were calculated according to the methods of Glower and colleagues. ${ }^{24}$ PRSW was based on linear regression of the relationship between SW and end-diastolic segment length calculated by the equation $\mathrm{SW}=\mathrm{M}_{\mathrm{sw}}$ (EDL-Lw), where $\mathrm{M}_{\mathrm{sw}}$ is the slope of PRSW and $\mathrm{L}_{\mathrm{w}}$ is the $\mathrm{x}$ axis intercept ${ }^{24}$ PRSWA was determined by the formula: PRSWA $=\mathrm{M}_{\mathrm{sw}} / 2\left(1.2 \mathrm{~L}_{\mathrm{w} \max }-\mathrm{L}_{\mathrm{w}}\right)^{2}$, where $\mathrm{L}_{\mathrm{w} \max }$ was the maximum $\mathrm{x}$-axis intercept during the entire experiment. Baseline and caval occlusion data were saved at specific time points in the protocol for off-line analysis. An average of 9 to 11 beats was used in each calculation.

Results are expressed as mean \pm SEM. Reperfusion segment shortening was expressed as percent recovery of preischemic values. Stroke work, $\mathrm{E}_{\mathrm{es}}, \mathrm{L}_{70}, \mathrm{PRSW}$, and PRSWA were expressed as absolute numbers.

Differences between the groups were determined with 2- factor analysis of variance with repeated measures across the second factor. If significant differences between groups were encountered, further analysis of variance was performed with Student $t$ tests. Differences within groups were analyzed with 1 -way analysis of variance.

\section{Results}

A total of 5 animals ( 4 controls and 1 preconditioned) were excluded because of ventricular fibrillation during ischemia or immediately on reperfusion. The systemic hemodynamic and global ventricular function data from the remaining 14 animals are summarized in Table I. There were no statistically significant differences in heart rate $(P=.97)$, mean arterial pressure $(P$ $=.33$ ), left ventricular developed pressure (LVDP; $P=$ $.20)$, left ventricle $+\mathrm{dP} / \mathrm{dt}(P=.40)$, or LAD blood flow $(P=.39)$ between the 2 groups at baseline or at anytime during the course of the reperfusion. The ischemic areas were similar in both groups $(25 \% \pm 3 \%$ of the left ventricle in the control group and $24 \% \pm 3 \%$ in the ischemic preconditioning group). There was no infarct in either group. Regional function in the left circumflex coronary beds in both groups remained stable throughout the experiment with the values of the percentage of segment shortening, SW, PRSW, and PRSWA at the conclusion of the 3-hour reperfusion, all being within $95 \%$ to $100 \%$ of baseline (data not shown).

Fig 1 shows the effects of the preconditioning protocol on regional segment shortening and SW. After the first and second cycles of ischemic preconditioning, LAD segment shortening was reduced from $19 \% \pm 2 \%$ to $15 \% \pm \%$ and $13 \% \pm 1 \%$, respectively (both values $P<.01$ vs baseline). In contrast, the preconditioning protocol did not cause any significant change in region- 


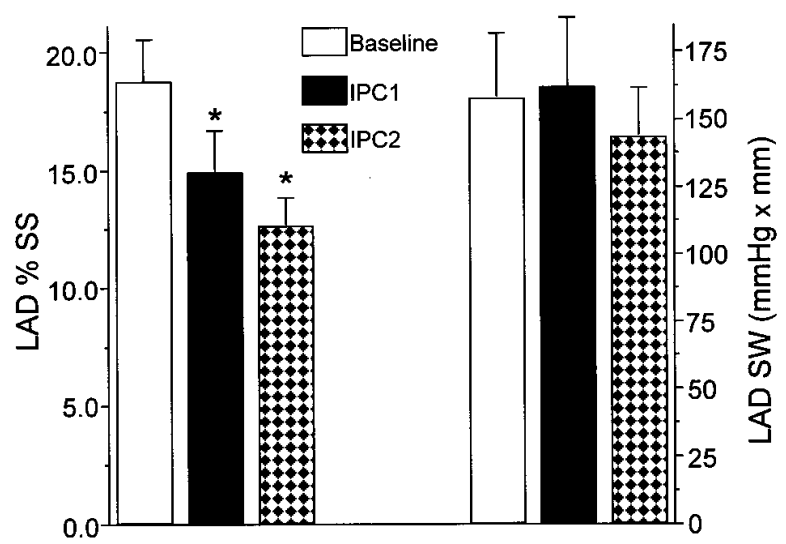

Fig 1. The effects of ischemic preconditioning (IPC) on baseline segment shortening (\%SS) and SW in the LAD bed. IPC1 and IPC2 refer to values obtained after 10 minutes of reperfusion after the first (IPC1) and second (IPC2) 5-minute LAD occlusions. $* P<.01$ versus baseline.

al SW. The normal baseline SW was $158 \pm 23 \mathrm{~mm} \mathrm{Hg}$ $\times \mathrm{mm}$; after the second preconditioning cycle, SW (reported as the baseline in Fig 3) was $144 \pm 18 \mathrm{~mm} \mathrm{Hg}$ $\times \mathrm{mm}(P=.16)$.

There was no statistically significant difference between the groups in baseline percent segment shortening $(P=.12)$. During ischemia, segment shortening decreased from $24 \% \pm 2 \%$ to $-4 \% \pm 2 \%$ in the control group; in the ischemic preconditioning group, segment shortening decreased from $13 \% \pm 1 \%$ to $-4 \% \pm 1 \%$. During reperfusion there was no statistically significant difference in the recovery of segment shortening between the 2 groups ( 1 hour, $P=.13 ; 2$ hours, $P=.10$; 3 hours, $P=.17$; Fig 2 ).

The effects of ischemic preconditioning on regional SW are shown in Fig 3. There were no statistically significant differences in SW between the 2 groups either at baseline $(P=.35)$ or at 2 hours $(P=.08)$ and 3 hours $(P=.14)$ of reperfusion. At 1 hour of reperfusion, SW was lower in the preconditioned group $(P=.01)$. Percent recovery of baseline regional SW after 3 hours of reperfusion was $50 \% \pm 6 \%$ in controls and $51 \% \pm$ $7 \%$ in preconditioned animals.

The ESPLR data collected during the caval occlusions are shown in Fig 4, $A$ and $B$. The reperfusion $\mathrm{E}_{\mathrm{es}}$ values were not significantly different between the groups at any time (1 hour, $P=.39 ; 2$ hours, $P=.27 ; 3$ hours, $P=.92$ ). Furthermore the reperfusion $\mathrm{E}_{\mathrm{es}}$ values were not significantly different from preischemic values within the groups (for controls 1-, 2-, and 3-hour $P$ values were $.88, .57$, and .44 , respectively, whereas for the preconditioned group they were $.27, .42$, and .18 ,

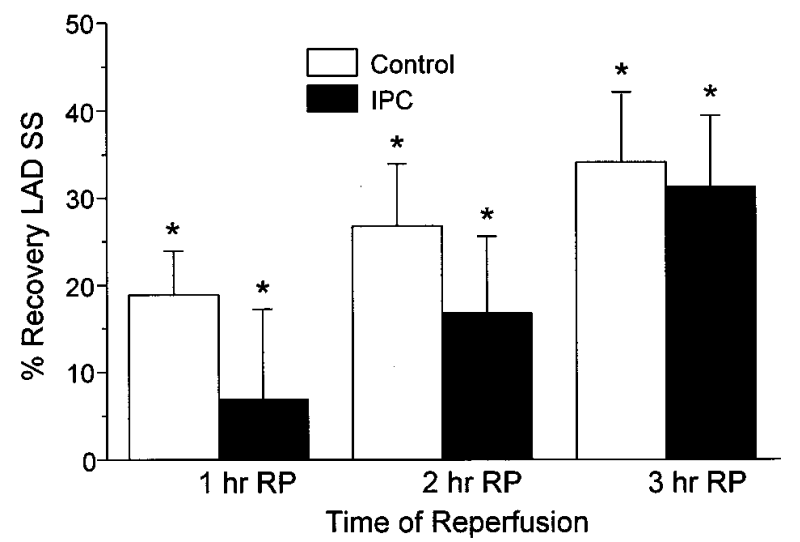

Fig 2. Recovery of segment shortening (SS) in control and ischemic preconditioning (IPC) groups after 15 minutes of LAD occlusion. Values are expressed as percent recovery of baseline segment shortening at 1,2, and 3 hours of reperfusion $(R P)$. The recovery of segment shortening in the IPC groups is expressed as percent of segment shortening values before the IPC protocol. All values are statistically different from preischemic values. $* P<.05$ versus baseline.

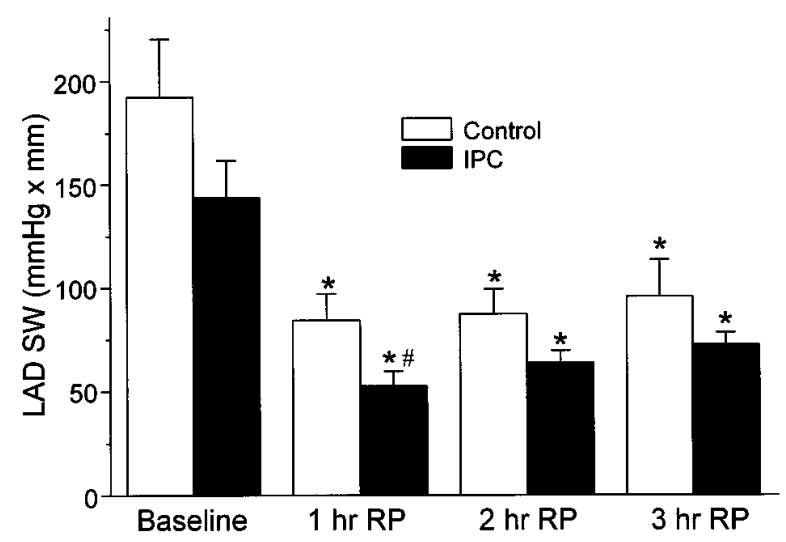

Fig 3. Baseline and reperfusion $(R P) \mathrm{SW}$ values in the LADperfused bed. The baseline SW in the ischemic preconditioning $(I P C)$ group is the value after the preconditioning occlusions but before the 15-minute ischemia (not significantly different from its baseline value, $P=.16$ ). ${ }^{*} P<.05$ versus baseline; $\# P=.01$ versus control group.

respectively). The control and preconditioned groups both exhibited significant rightward-shifts in ESPLR throughout the 3-hour reperfusion period (Fig 4, B). Although reperfusion $\mathrm{L}_{70}$ values tended to be higher in preconditioned hearts, there were no statistically significant differences between the groups (1-, 2-, and 3hour $P$ values were $.08, .14$, and .14 , respectively).

The effects of ischemia on PRSW are shown in Fig 5, 

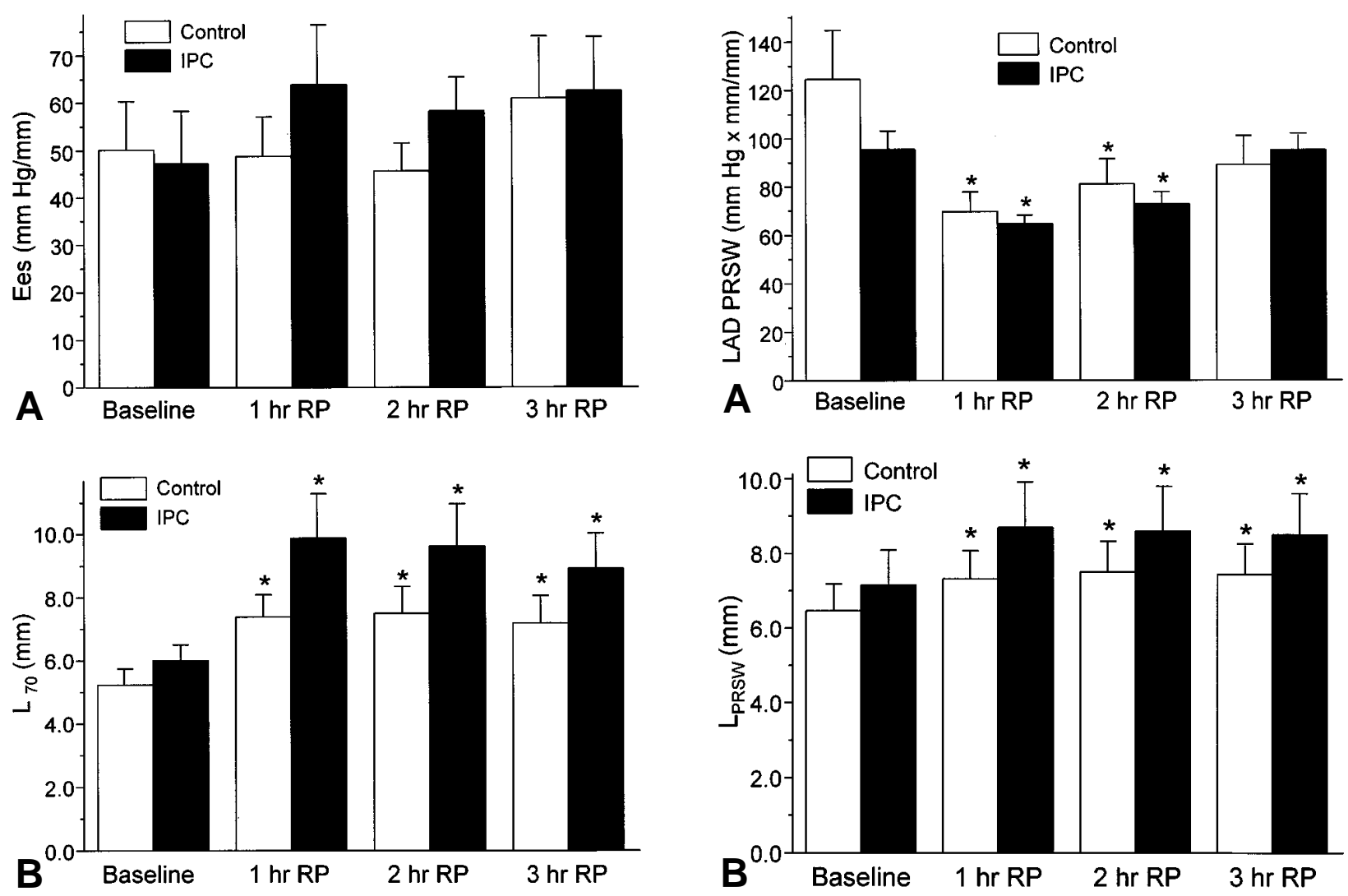

Fig 4. Values of the slope (Ees; $\mathbf{A})$ and length axis intercept at a pressure of $70 \mathrm{~mm} \mathrm{Hg}\left(L_{70} ; \mathbf{B}\right)$ in control and ischemic preconditioned pigs. $\mathrm{E}_{\mathrm{es}}$ and $\mathrm{L}_{70}$ were calculated from the ESPLR generated during brief vena cava occlusions. ${ }^{*} P<.05$ versus baseline.

$A$ and $B$. The preischemic PRSW values were similar in both groups $(P=.10)$. The percent recovery of PRSW in the control group at 1-, 2-, and 3-hour reperfusion was $61 \% \pm 9 \%, 70 \% \pm 10 \%$, and $75 \% \pm 10 \%$, respectively. Ischemic preconditioning was associated with similar effects on reperfusion PRSW, and the percent recovery in this group at 1-, 2-, and 3-hour reperfusion was $70 \% \pm 5 \%, 78 \% \pm 6 \%$, and $102 \% \pm 9 \%$, respectively (1-, 2-, and 3-hour $P$ values vs control were .36, .42 , and .08 , respectively). Fig $5, B$, shows the preischemic and reperfusion PRSW x-intercept values in the 2 groups, indicating both groups exhibited significant rightward shifts throughout reperfusion compared with baseline values. But there was no statistically significant difference between the groups (1-, 2-, and 3-hour $P$ values vs control were $.24, .35$, and .31 , respectively).

The effects of ischemia and the ischemic preconditioning protocol on PRSWA are shown in Fig 6. There was no statistically significant difference between the

Fig 5. Effects of ischemic preconditioning (IPC) on PRSW in stunned myocardium. PRSW is based on the slope (A) and length axis intercept $\left(L_{P R S W} ; \mathbf{B}\right)$ of PRSW calculated from data generated during brief vena cava occlusions. ${ }^{*} P<.05$ versus baseline.

baseline PRSWA values between the 2 groups $(P=$ $.87)$, and they remained significantly depressed throughout reperfusion. After 3 hours of reperfusion, the recovery of PRSWA in the control group was $48 \%$ $\pm 8 \%$ of baseline, and in the preconditioned group it was $62 \% \pm 4 \%$ of baseline. There was no statistically significant difference between the 2 groups at any time during reperfusion ((1-, 2-, and 3-hour $P$ values vs control were $.82, .43$, and .30 , respectively).

\section{Discussion}

The results of this study confirm that ischemic preconditioning does not attenuate regional myocardial stunning when assessed by segment shortening. Furthermore ischemic preconditioning does not improve regional SW, or cardiac contractility, assessed by the relatively load-insensitive measures of ESPLR, PRSW, and PRSWA. Hence, these results indicate that ischemic preconditioning does not acutely improve car- 
diac contractility in in vivo regionally stunned porcine myocardium.

Several experimental and clinical studies have suggested that ischemic preconditioning reduces myocardial infarct size and ventricular arrhythmias, but its effects on postischemic ventricular function are less clear. Numerous studies show that globally ischemic isolated perfused rat hearts can be preconditioned, ${ }^{9-11}$ and there are similar studies in isolated rabbit hearts. ${ }^{12-14}$ However, additional reports in isolated rabbit $^{15,16}$ and guinea pig hearts ${ }^{4}$ show that ischemic preconditioning does not improve postischemic function. Furthermore, high creatine kinase and lactate dehydrogenase release during reperfusion and high postischemic end-diastolic pressures in the isolated rat heart studies indicate that there was significant infarct. Jenkins and colleagues ${ }^{17}$ measured both function and infarct size in globally ischemic isolated rabbit hearts and reported that ischemic preconditioning improved postischemic LVDP when infarct size was reduced, but preconditioning did not attenuate pure stunning in the absence of infarct.

Although there are numerous studies of ischemic preconditioning effects on postischemic function in isolated hearts, there are only a few studies in in vivo pure myocardial stunning models. Cohen and colleagues ${ }^{18}$ reported that preconditioning improved segment shortening in the rabbit after 20 minutes of regional ischemia; however, they attributed this improvement to the associated $25 \%$ decrease in infarct size. Two frequently cited studies reported that neither a single cycle (Ovize and colleagues ${ }^{19}$ ) nor 2 cycles (Miyamae and colleagues $^{20}$ ) of 5-minute preconditioning occlusions improve segment shortening after 15 minutes of regional ischemia in the canine or porcine model, respectively. However, any protective effect of the preconditioning may have been masked by the fact that the 5-minute preconditioning cycles reduced segment shortening considerably (52\% and $25 \%$, respectively) before the prolonged occlusion.

Our results are consistent with those of Ovize, ${ }^{19}$ Miyamae, ${ }^{20}$ and their colleagues in terms of the lack of beneficial effect of ischemic preconditioning on postischemic regional ventricular function in the absence of myocardial infarction. We also observed a significant decrease in segment shortening after the two 5-minute preconditioning occlusions. In all of these studies, the stunning produced by the preconditioning occlusions, which likely would have persisted for several hours, confounds the interpretation of the potential beneficial effects of preconditioning on postischemic function. The present results with regional stroke work are less

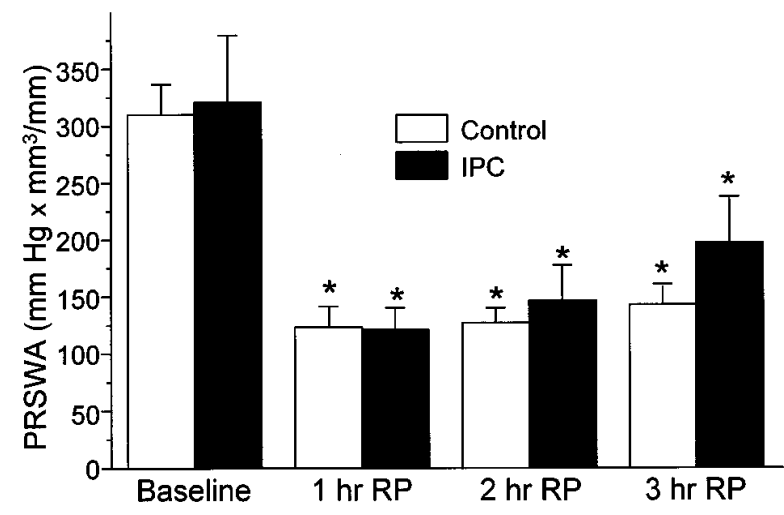

Fig 6. PRSWA in the LAD-perfused bed in control and ischemic preconditioned (IPC) groups. PRSWA was calculated as described by Glower and colleagues. ${ }^{24} * P<.05$ versus baseline.

problematic to interpret. The 2 preconditioning cycles did not significantly reduce regional SW before the long occlusion, nor did this protocol improve regional SW after the prolonged occlusion. Because segment shortening is both preload and afterload sensitive and SW is preload sensitive, the use of these measures as reliable assessments of cardiac contractility is limited.

The novel aspect to the present study was our analysis of the effects of ischemic preconditioning on stunning measured by ESPLR, PRSW, and PRSWA. All of these measures of cardiac contractility are relatively load insensitive. ${ }^{23,24} \mathrm{E}_{\mathrm{es}}$ is the slope of the ESPLR generated during brief vena cava occlusions and is analogous to end systolic elastance $\left(\mathrm{E}_{\mathrm{es}}\right)$ of ESPVR. ${ }^{25}$ Aversano and colleagues ${ }^{23}$ first reported the reliability of ESPLR for measurements of regional contractility in an open chest preparation. In the present study, although $\mathrm{E}_{\mathrm{es}}$ was not significantly altered with stunning, the ESPLR in both groups was rightward shifted throughout reperfusion, as indicated by significantly greater $\mathrm{L}_{70}$ values; there were no statistical differences between the groups. Thus preconditioning did not attenuate stunning when assessed by ESPLR.

Another load-insensitive measure of myocardial contractility is the PRSW. This index of contractility is the relationship between regional stroke work and EDL and has been shown to be linear and a load-insensitive measure of regional inotropic state in both normal and reperfused myocardium. ${ }^{24} \mathrm{~A}$ decrease in contractility based on PRSW is exhibited by a decrease in the slope and an increase in the length axis intercept. In our study, the PRSW slopes after 3-hour reperfusion in both groups returned to values that were not statistically different from preischemic values. However, the 
regression lines in both groups were significantly rightward shifted throughout reperfusion, indicating persistent stunning consistent with the results of Glower and colleagues. ${ }^{24}$

PRSWA has been reported to be the most sensitive for quantifying regional ischemia-induced ventricular dysfunction. ${ }^{24}$ In our study, this index of regional contractility remained significantly reduced in both groups throughout reperfusion, indicating persistent myocardial stunning. Furthermore, the lack of statistical difference in the recovery of PRSWA between the 2 groups indicates that ischemic preconditioning did not improve this load-insensitive measure of contractility.

There are several limitations to the present study, which must be recognized. These results pertain only to the first 3 hours of reperfusion and do not reflect the potential benefits during the reperfusion beyond 3 hours. Although we observed no significant differences between the groups, it appeared that there was a slight trend for improved recovery of PRSWA in the ischemic preconditioned group at the conclusion of the study. This trend may have translated into a statistically significant difference if the reperfusion period had been longer. Another limitation is the sample size. Although the sample size of each group was 7, the study had sufficient power to detect a difference between the groups. It is also possible that ischemic preconditioning may have exerted a beneficial effect after a longer ischemic period. We limited the ischemia to 15 minutes to avoid the development of infarction because a 15-minute occlusion has been repeatedly shown to result in no infarct in either porcine or canine myocardium. ${ }^{19,20} \mathrm{We}$ did not see any infarction using triphenyltetrazolium chloride staining, which is consistent with these studies. The use of an open chest, pentobarbital anesthetized animal preparation may have influenced both the degree of ischemic injury and the precision of the load-insensitive measurements. Finally, it remains to be determined whether these findings in a regional ischemia model can be extrapolated to the setting of global ischemia.

Our findings are consistent with Cremer and colleagues,${ }^{27}$ who compared a group of patients receiving a preconditioning protocol identical to ours before arrest with cold blood cardioplegia versus patients receiving intermittent cold blood cardioplegia alone and found no benefit from ischemic preconditioning. There are other studies that report no beneficial effects of ischemic preconditioning during clinical cardiac operation. ${ }^{28-30}$

In summary, the results of this study indicate that ischemic preconditioning does not ameliorate porcine myocardial stunning in the acute reperfusion period, when contractility is assessed by load-insensitive measurements. Extrapolating these results to the clinical arena ischemic preconditioning may have a limited role in minimizing reversible postischemic dysfunction after cardiac operation.

We thank Kati Kraft and Elizabeth Partin for excellent technical assistance.

\section{REFERENCES}

1. Murry CE, Jennings RB, Reimer KA. Preconditioning with ischemia: a delay of lethal cell injury in ischemic myocardium. Circulation 1986;74:1124-36.

2. Pryzklenk K, Kloner RA. Ischemic preconditioning: exploring the paradox. Prog Cardiovasc Dis 1998;40:517-47.

3. Murry CE, Richard VJ, Reimer KA, Jennings RB. Ischemic preconditioning slows energy metabolism and delays ultrastructural damage during a sustained ischemic episode. Circ Res 1990;66: 913-31.

4. Steenbergen C, Perlman ME, London RE, Murphy E. Mechanism of preconditioning: ionic alterations. Circ Res 1993; 72:112-25.

5. Van Wylen DGL. Effect of ischemic preconditioning on interstitial purine metabolite and lactate accumulation during myocardial ischemia. Circulation 1994;89:2283-9.

6. Lindhardt TB, Kelbaek H, Madsen JK, Saunamaki K, Clemmensen $\mathrm{P}$, Hesse B, et al. Continuous monitoring of global left ventricular ejection fraction during percutaneous transluminal coronary angioplasty. Am J Cardiol 1998;81:853-9.

7. Lu EX, Chen SX, Hu TH, Xui LM, Yuan MD. Preconditioning enhances myocardial protection in patients undergoing open heart surgery. Thorac Cardiovasc Surg 1998;46:28-32.

8. Alkhulaifi AM, Yellon DM, Pugsley WB. Preconditioning the human heart during aorto-coronary bypass surgery. Eur $\mathrm{J}$ Cardiothorac Surg 1994;8:270-6.

9. Asimakis GK, Inners-McBride K, Conti VR. Attenuation of postischemic dysfunction by ischemic preconditioning is not mediated by adenosine in isolated rat heart. Cardiovasc Res 1993; 27:1522-30.

10. Cave AC, Collis CS, Downey JM, Hearse DJ. Improved functional recovery by ischemic preconditioning is not mediated by adenosine in the globally ischemic isolated rat heart. Cardiovasc Res 1993;27:663-8.

11. Lasley RD, Anderson GM, Mentzer RM Jr. Ischemic and hypoxic preconditioning enhance postischemic recovery of function in the rat heart. Cardiovasc Res 1993;27:565-70.

12. Hendrikx M, Toshima Y, Mubagwa K, Flameng W. Improved functional recovery after ischemic preconditioning in the globally ischemic rabbit heart is not mediated by adenosine $A_{1}$ receptor activation. Basic Res Cardiol 1993;88:576-93.

13. Bolling SF, Olszanski DA, Childs KF, Gallagher KP, Ning XH. Stunning, preconditioning, and functional recovery after global myocardial ischemia. Ann Thorac Surg 1994;58:822-7.

14. Illes RW, Wright JK, Inners-McBride K, Yang CJ, Tristan A. Ischemic preconditioning improves preservation with crystalloid cardioplegia. Ann Thorac Surg 1994;58:1481-5.

15. Sandhu R, Diaz RJ, Wilson GJ. Comparison of ischemic precon- 
ditioning in blood perfused and buffer perfused isolated heart models. Cardiovasc Res 1993;27:602-7.

16. Lasley RD, Noble MA, Konyn PJ, Mentzer RM Jr. Different effects of an adenosine $A_{1}$ analogue and ischemic preconditioning in isolated rabbit hearts. Ann Thorac Surg 1995;60:1698-703.

17. Jenkins DP, Pugsley WB, Yellon DM. Ischemic preconditioning in a model of global ischemia: infarct size limitation, but no reduction of stunning. J Mol Cell Cardiol 1995;27:1623-32.

18. Cohen MV, Liu GS, Downey JM. Preconditioning causes improved wall motion as well as smaller infarcts after transient coronary occlusion in rabbits. Circulation 1991;84:341-9.

19. Ovize M, Pryzklenk K, Hale SL, Kloner RA. Preconditioning does not attenuate myocardial stunning. Circulation 1992;85: 2247-54.

20. Miyamae M, Fujiwara H, Kida M, Yokota R, Tanaka M, Katsuragawa M, et al. Preconditioning improves energy metabolism during reperfusion but does not attenuate myocardial stunning in porcine hearts. Circulation 1993;88:223-34.

21. Pagel PS, Kampine JP, Schmelling W, Warltier D. Comparison of end systolic pressure-length relations and preload recruitable stroke work as indices of myocardial contractility in the conscious and anesthetized chronically instrumented dog. Anesthesiology 1990;73:278-90.

22. Foex P, Francis CM, Cutfield GR, Leone B. The pressure length loop. Br J Anaesth 1988;60:65S-71S.

23. Aversano T, Maughan WL, Hunter WC, Kass D, Becker L. Endsystolic measures of regional ventricular performance. Circulation 1986;73:5:938-50.
24. Glower DD, Spratt JA, Snow ND, Kabas JS, Davis Rankin JS. Linearity of the Frank-Starling relationship in the intact heart: the concept of preload recruitable stroke work. Circulation 1985; 71:994-1009.

25. Suga H, Sagawa K, Kostiuk DP. Controls of ventricular contractility assessed by pressure-volume ratio, $\mathrm{E}_{\max }$. Cardiovasc Res 1976;10:582-92.

26. Faris B, Peynet J, Wassef M, Bel A, Mouas C, Duriez M, et al. Failure of preconditioning to improve post cardioplegia stunning of minimally infarcted hearts. Ann Thorac Surg 1997;64:173541.

27. Cremer J, Steinhoff G, Karck M, Ahnsell T, Brandt M, Teebken $\mathrm{OE}$, et al. Ischemic preconditioning prior to myocardial protection with cold blood cardioplegia in coronary surgery. Eur J Cardiothorac Surg 1997;12:753-8.

28. Malkowski MJ, Kramer CM, Parvizi ST, Dianzumba S, Marquez J, Reichek N, et al. Transient ischemia does not limit subsequent ischemic regional dysfunction in humans: a transesophageal echocardiographic study during minimally invasive coronary artery bypass surgery. J Am Coll Cardiol 1998;5: $1035-9$.

29. Kaukoranta PK, Lepojarvi MP, Ylitalo KV, Kiviluoma KT, Peuhkurinen KJ. Normothermic retrograde blood cardioplegia with or without preceding ischemic preconditioning. Ann Thorac Surg 1997;63:1268-74.

30. Perrault LP, Menasche P, Bel A, de Chaumaray T, Peynet J, Mondry A, et al. Ischemic preconditioning in cardiac surgery: a word of caution. J Thorac Cardiovasc Surg 1996;112:1378-86. 